\title{
Sildenafil no tratamento da hipertensão arterial pulmonar idiopática em crianças e adolescentes
}

\author{
Sildenafil in the management of idiopathic pulmonary arterial hypertension \\ in children and adolescents
}

Edmundo Clarindo Oliveira', Carlos Faria Santos Amaral'

\section{Resumo}

Objetivo: Este artigo tem por objetivo relatar a experiência com o uso do sildenafil oral no tratamento de pacientes com hipertensão arterial pulmonar idiopática grave em classe funcional III e IV, sem resposta à terapia convencional e às provas de reatividade pulmonar.

Método: Trata-se de estudo prospectivo, tipo de série de casos em que seis pacientes com diagnóstico de hipertensão arterial pulmonar idiopática foram tratados com sildenafil oral, com doses de 2 a $8 \mathrm{mg} /$ $\mathrm{kg} / \mathrm{dia}$, divididas em quatro a seis vezes. Os pacientes foram submetidos a exame clínico, eletrocardiograma, ecocardiograma, tomografia do tórax, cintilografia de perfusão e ventilação pulmonar, estudos da coagulação, pesquisa de esquistossomose, doença do colágeno e de síndrome de imunodeficiência adquirida para afastar causas secundárias de hipertensão pulmonar. Todos os pacientes foram submetidos a cateterismo cardíaco para a realização das provas de reatividade pulmonar com óxido nítrico, $\mathrm{O}_{2}$ a $100 \%$ e nifedipina oral e ao teste de caminhada de 6 minutos nos pacientes em condições de realizá-lo.

Resultados: Todos eles apresentaram boa resposta, traduzida por melhora de, pelo menos, uma classe funcional, aumento da saturação sistêmica. Cinco pacientes apresentaram diminuição da relação da pressão sistólica pulmonar/pressão sistólica sistêmica e aumento da distância percorrida no teste de caminhada nos que a realizaram. Não foram observados efeitos colaterais em um tempo de seguimento de 4 a 36 meses. Um paciente apresentou morte súbita após a suspensão do medicamento.

Conclusões: Os resultados sugerem que o sildenafil possa ser uma droga promissora no tratamento dos pacientes com hipertensão pulmonar idiopática, os quais devem ser conscientizados do risco da suspensão da medicação sem orientação médica.

J Pediatr (Rio J). 2005;81(5):390-4: Hipertensão arterial pulmonar; inibidores da fosfodiesterase.

\section{Abstract}

Objective: This study aims to provide data on the use of oral sildenafil in patients in New York Heart Association functional class III or IV with severe idiopathic pulmonary arterial hypertension unresponsive to conventional therapy.

Method: In this series, six patients with idiopathic pulmonary arterial hypertension were prospectively treated with 2 to $8 \mathrm{mg}$ of oral sildenafil in four to six doses a day. All patients were submitted to physical examination, electrocardiogram and echocardiogram, chest computed tomography, ventilation and pulmonary perfusion scintigraphy, coagulation studies, and tests for collagen vascular disease, acquired immune deficiency syndrome and schistosomiasis in order to rule out secondary causes of pulmonary arterial hypertension. All patients underwent cardiac catheterization for vasoreactivity tests using nitric oxide, $\mathrm{O}_{2}$ at $100 \%$ and oral nifedipine, and a 6 -minute walking test was performed in those patients who were considered able to exercise.

Results: All patients achieved a good therapeutic response, with improvement by at least one functional class, and presented an increase in systemic arterial oxygen saturation. Five patients showed a decrease in the pulmonary systolic pressure to systemic systolic pressure ratio and improvement in the six-minute walking test. No major side effects were observed at 4 to 36 months of follow-up. One patient had sudden death after sildenafil had been withdrawn by mistake.

Conclusions: These data suggest that sildenafil may be useful in the management of idiopathic pulmonary arterial hypertension. Patients should be advised against the withdrawal of sidenafil without medical supervision.

J Pediatr (Rio J). 2005;81(5):390-4: Pulmonary arterial hypertension, phosphodiesterase inhibitors.

1. Chefe do serviço de cardiologia, Hospital Vera Cruz. Chefe do serviço de cardiologia pediátrica da Fundação Hospitalar de Minas Gerais. Hemodinamicista pediátrico, Hospital das Clínicas, Universidade Federal de Minas Gerais (UFMG), Belo Horizonte, MG.

2. Doutor. Professor adjunto, UFMG, Belo Horizonte, MG.

Artigo submetido em 03.02.05, aceito em 11.05.05.

Como citar este artigo: Oliveira EC, Amaral CF. Sildenafil no tratamento da hipertensão arterial pulmonar idiopática em crianças e adolescentes. J Pediatr (Rio J). 2005;81:390-4.

\section{Introdução}

A hipertensão arterial pulmonar idiopática (HAPI) é definida como pressão média na artéria pulmonar acima de $25 \mathrm{mmHg}$ em repouso e $30 \mathrm{mmHg}$ sob esforço, de etiologia indeterminada e com pressão capilar pulmonar normal ${ }^{1}$. Trata-se de doença rara, com uma incidência de um a dois casos/milhão de habitantes/ano. A HAPI pode apresentar predisposição familiar em $10 \%$ dos casos. 
Observações sugerem que a vasoconstrição tem um papel importante na patogenia dessa doença, que é caracterizada por hipertrofia patológica da camada média das arteríolas pulmonares, diminuição da produção endotelial dos vasodilatadores prostaciclina e óxido nítrico e aumento da produção do vasoconstritor endotelina ${ }^{2}$. Os sintomas, como cansaço, tonteira, dor torácica, insuficiência cardíaca e hipoxemia, usualmente aparecem quando a hipertensão é grave. A partir do seu início, a expectativa é de 68,48 e $34 \%$ em 1,3 e 5 anos, respectivamente ${ }^{3}$, na ausência de tratamento para diminuir a pressão pulmonar (PP). A doença não tem cura, e o paciente necessita de tratamento por toda a vida. A diminuição da PP é a forma mais eficiente de promover o alívio dos sintomas de insuficiência cardíaca, aumentar a sobrevida e melhorar a qualidade de vida desses pacientes. Infelizmente, não existe uma droga ideal para essa finalidade.

Várias drogas têm sido usadas de modo contínuo, sendo os antagonistas de cálcio, principalmente a nifedipina, os que vêm sendo empregados há mais tempo. A ação vasodilatadora pulmonar desses antagonistas é reconhecida, com diminuição da PP e aumento da sobrevida dos pacientes com HAPI ${ }^{4}$. As doses necessárias para a redução da PP são usualmente maiores do que as empregadas no tratamento da hipertensão arterial sistêmica. Sabe-se, também, que a maioria dos pacientes não responde aos antagonistas de cálcio, podendo apresentar efeitos colaterais graves, o que torna fundamental a realização prévia de provas de reatividade pulmonar para selecionar aqueles que, de fato, se beneficiariam com o seu uso. As provas de reatividade pulmonar são feitas com oxigênio a $100 \%$ e drogas de meiavida curta com ação vasodilatadora pulmonar, como a adenosina $^{5}$, a prostaciclina 6 , a acetilcolina e, principalmente, o óxido nítrico ${ }^{7}$, por ser um vasodilatador pulmonar seletivo e de curta ação.

As prostaglandinas ${ }^{8}$, em forma de aerossol ou bomba de infusão contínua, são drogas promissoras, mas apresentam alguns inconvenientes, como a necessidade de uso em curtos intervalos, o risco de complicações (infecção e trombose) quando usadas em infusão contínua e o custo elevado. Os inibidores da endotelina ${ }^{9}$ têm sido recentemente investigados, mas também são de custo elevado e sujeitos a efeitos colaterais graves, como alteração hepática. Outras medidas, como o uso de oxigênio domiciliar e anticoagulantes orais, são apenas paliativas, e seu impacto no aumento da sobrevida dos pacientes com HAPI não está definido. A atriosseptostomia $^{10}$ e o transplante pulmonar ${ }^{11}$ constituem formas extremas de tratamento da HAPI e apresentam um resultado ruim a médio prazo.

Mais recentemente, o sildenafil (Viagra ${ }^{\circledR}$ ), usado no tratamento da disfunção erétil e comercializado para esse fim, passou a ser utilizado no tratamento da HAPI. A droga é um inibidor seletivo e potente da fosfodiesterase 5 (PDE5) específica para o monofosfato de guanosina cíclico (GMPc), abundante nos pulmões. A inibição da PDE5 impede a degradação de GMPc, que é um mensageiro intracelular do óxido nítrico, com conseqüente vasodilatação pulmonar. A literatura tem apresentado relatos progressivamente mais freqüentes sobre o uso do sildenafil para o tratamento da hipertensão pulmonar (HP) de diversas causas ${ }^{12,13}$.

Este trabalho relata os resultados do tratamento com sildenafil oral de seis pacientes com HAPI grave em classe funcional III e IV, a despeito do uso de medidas terapêuticas convencionais.

\section{Casuística e método}

Trata-se de estudo do tipo série de casos, prospectivo, envolvendo seis pacientes com diagnóstico de hipertensão pulmonar idiopática grave, refratários ao tratamento convencional. O diagnóstico de HP foi suspeitado pelo aumento da intensidade do componente pulmonar da segunda bulha à ausculta e pelos sinais de aumento do ventrículo direito (VD), avaliado pela radiografia de tórax e pelo eletrocardiograma. As alterações ecocardiográficas para o diagnóstico de HP incluíram aumento da PP, utilizando-se o gradiente ventrículo direito-átrio direito (VD-AD), estimado pela regurgitação tricúspide e acrescido de $10 \mathrm{mmHg}$, valor este assumido como pressão de átrio direito $(A D)$. Causas secundárias de HP foram afastadas por meio de exames de tomografia torácica, cintilografia pulmonar de ventilaçãoperfusão, estudo da coagulação, pesquisa de esquistossomose e de síndrome de imunodeficiência adquirida, ecocardiograma e cateterismo cardíaco. Para a realização do cateterismo cardíaco, todos os pacientes foram sedados com midazolan e fentanil.

A monitorização da PP foi feita com cateter de SwanGanz 7F para adultos e $5 \mathrm{~F}$ para crianças e da pressão arterial (PA) com cateter $3 \mathrm{~F}$ inserido na artéria femoral. Durante o cateterismo, foram feitas medidas das pressões da artéria pulmonar, aorta, capilar pulmonar e do AD. Foram realizadas determinações do débito cardíaco e das resistências vasculares pulmonar e sistêmica. Essas medidas foram feitas com o paciente respirando ar ambiente, após uso de oxigênio a $100 \%$ por 15 minutos e de óxido nítrico em concentração inicial de $10 \mathrm{ppm}$. Na falta de resposta, essa concentração foi aumentada, a cada 10 minutos, até 80 $\mathrm{ppm}^{6}$. A nifedipina oral foi administrada na dose inicial de 5 $\mathrm{mg}$ para pacientes acima de $30 \mathrm{~kg}$ e de $0,2 \mathrm{mg} / \mathrm{kg}$ para crianças, com aumento de $50 \%$ da dose, a cada 6 horas, na ausência de resposta ou de efeitos colaterais. As provas de reatividade pulmonar foram definidas como positivas na ocorrência de diminuição da PP média maior que $20 \%$, sem diminuição do débito sistêmico e com manutenção da redução da relação pressão pulmonar sistólica (PPS)/pressão arterial sistólica (PAS) maior que $20 \%$. Diminuição do débito sistêmico, manutenção ou aumento da relação PPS/PAS, taquicardia persistente e diminuição na saturação sistêmica de oxigênio foram consideradas como falta de resposta às provas. Os pacientes foram observados em CTI, com monitorização da PP e da PA por 12 a 24 horas. O teste da caminhada de 6 minutos não foi realizado em quatro pacientes, por estarem em uso de drogas vasoativas em CTI, com insuficiência cardíaca e hipoxemia grave e/ou serem crianças. As medicações em uso foram mantidas. O sildenafil oral foi usado em dose inicial de $2 \mathrm{mg} / \mathrm{kg} / \mathrm{dia}$, até o máximo de $100 \mathrm{mg} /$ dia, divididos em quatro ou seis doses. 
Foi estabelecido que as doses poderiam ser aumentadas, a cada 15 dias, até $8 \mathrm{mg} / \mathrm{kg} / \mathrm{dia}$, ou até atingir $500 \mathrm{mg} / \mathrm{dia}^{14}$, na falta de resposta ou de efeitos colaterais.

O protocolo de pesquisa foi aprovado pelo Comitê de Ética do Hospital Vera Cruz de Belo Horizonte e pelo Comitê de Ética em Pesquisa da Universidade Federal de Minas Gerais (UFMG). A inclusão deu-se após assinatura do Termo de Consentimento Livre e Esclarecido (TCLE) pelos pacientes e/ou seus representantes legais, no qual constava a informação de que o sildenafil estava sendo testado para tratamento da HAPI e que não era comercializado primariamente para esse fim. Foram disponibilizadas facilidades para contato dos pacientes e seus familiares com o médico assistente para tratamento de intercorrências.

\section{Resultados}

As características clínicas dos seis pacientes encontram-se na Tabela 1. Todos estavam em classe funcional III ou IV, mesmo em uso de medicação para tratamento da insuficiência cardíaca, incluindo o uso de dopamina em dois pacientes. Nas Tabelas 2 e 3, estão os resultados dos exames complementares realizados. Os dados apresentados nessas tabelas mostram que todos os pacientes tinham pressão capilar pulmonar normal. Cinco dos seis pacientes apresentavam pressões pulmonares supra-sistêmicas, e o outro paciente em níveis sistêmicos. A saturação sistêmica de oxigênio era inferior a $90 \%$ em quatro deles, e nenhum paciente teve resposta aos testes de reatividade pulmonar. $\mathrm{Na}$ Tabela 4, encontram-se descritos os resultados e a evolução clínica após administração oral do sildenafil. No período de seguimento, observou-se melhora de, pelo menos, uma classe funcional em todos os pacientes, melhora da saturação sistêmica de oxigênio e aumento da distância percorrida em 6 minutos nos pacientes submetidos ao teste de caminhada. Cinco dos seis pacientes apresentaram queda da relação PPS/PAS, traduzindo uma diminuição efetiva da PP.

\section{Discussão}

Os seis pacientes incluídos neste estudo apresentaram melhora clínica após o uso do sildenafil oral, traduzida por redução de, pelo menos, uma classe funcional da NYHA

Tabela 1 - Características clínicas dos seis pacientes com hipertensão arterial pulmonar idiopática

\begin{tabular}{|c|c|c|c|c|c|c|c|c|c|c|c|c|c|c|}
\hline \multirow[t]{2}{*}{ Paciente } & \multirow{2}{*}{$\begin{array}{l}\text { Idade } \\
\text { (anos) }\end{array}$} & \multirow[t]{2}{*}{ Sexo } & \multirow{2}{*}{$\begin{array}{c}\text { CF } \\
\text { (NYHA) }\end{array}$} & \multirow[t]{2}{*}{$\uparrow \mathbf{B}_{2}$} & \multirow[t]{2}{*}{ IT } & \multirow[t]{2}{*}{ HM } & \multicolumn{5}{|c|}{ Medicação } & \multirow[t]{2}{*}{ CTI } & \multirow[t]{2}{*}{ ENF } & \multirow[t]{2}{*}{ AMB } \\
\hline & & & & & & & Dig & Warf & Diur & $\mathbf{O}_{2}$ & Dob & & & \\
\hline 1 & 4,5 & $\mathrm{~F}$ & IV & + & + & + & + & + & + & + & + & + & - & - \\
\hline 2 & 11 & $\mathrm{~F}$ & III & + & + & - & - & + & - & - & - & - & - & + \\
\hline 3 & 3 & $\mathrm{~F}$ & IV & + & + & + & + & + & + & + & + & + & - & - \\
\hline 4 & 19 & $\mathrm{~F}$ & IV & + & + & + & + & + & + & + & - & - & + & - \\
\hline 5 & 19 & $\mathrm{~F}$ & III & + & + & - & + & + & - & - & - & - & - & + \\
\hline 6 & 4 & $M$ & IV & + & + & + & + & + & + & + & - & - & + & - \\
\hline
\end{tabular}

$\mathrm{CF}=$ classe funcional; NYHA = New York Heart Association; $\uparrow \mathrm{B}_{2}=$ hiperfonese de $\mathrm{B}_{2} ; \mathrm{IT}=$ insuficiência tricúspide; $\mathrm{HM}=$ hepatomegalia; Dig = Digital; Warf = Warfarina, Diur = Diurético; $\mathrm{O}_{2}=$ oxigenoterapia; $\mathrm{Dob}=$ Dobutamina; $\mathrm{CTI}=$ centro de tratamento intensivo; $\mathrm{ENF}=$ enfermaria; $\mathrm{AMB}=\mathrm{ambulatório} ;(+)=\mathrm{presente} ;$ $(-)=$ ausente.

Tabela 2 - Resultados dos exames complementares realizados em seis pacientes com hipertensão arterial pulmonar idiopática

\begin{tabular}{|c|c|c|c|c|c|c|c|c|}
\hline \multirow[t]{2}{*}{ Paciente } & \multirow[t]{2}{*}{ ECG } & \multirow[t]{2}{*}{$\begin{array}{l}\text { Área cardíaca } \\
\text { (Raio-x } \\
\text { do tórax) }\end{array}$} & \multicolumn{2}{|c|}{$\begin{array}{c}\text { ECG } \\
\text { Função ventricular } \\
(\downarrow, \mathbf{N})\end{array}$} & \multirow[t]{2}{*}{$\begin{array}{c}\text { PSAP* } \\
(\mathbf{m m H g})\end{array}$} & \multirow[t]{2}{*}{$\begin{array}{c}\text { PAS } \\
(\mathrm{mmHg})\end{array}$} & \multirow[t]{2}{*}{ PPS/PAS } & \multirow[t]{2}{*}{$\begin{array}{l}\mathrm{SpO}_{2} \\
(\%)\end{array}$} \\
\hline & & & VD & VE & & & & \\
\hline 1 & SVD & $\uparrow$ & $\downarrow$ & $\mathrm{N}$ & 105 & $80 / 60$ & 1,31 & 90 \\
\hline 2 & SVD & $\mathrm{N}$ & $\mathrm{N}$ & $\mathrm{N}$ & 90 & $100 / 70$ & 0,90 & 98 \\
\hline 3 & SVD & $\uparrow$ & $\downarrow$ & $\mathrm{N}$ & 110 & 90 & 1,22 & 85 \\
\hline 4 & SVD & $\uparrow$ & $\downarrow$ & $\mathrm{N}$ & 110 & 95 & 1,16 & 88 \\
\hline 5 & SVD & $\uparrow$ & $\mathrm{N}$ & $\mathrm{N}$ & 120 & $100 / 60$ & 1,20 & 86 \\
\hline 6 & SVD & $\uparrow$ & $\downarrow$ & $\mathrm{N}$ & 105 & $90 / 70$ & 1,17 & 74 \\
\hline
\end{tabular}

$\overline{E C G}=$ ecocardiograma; VD = ventrículo direito; VE = ventrículo esquerdo; PSAP* = pressão sistólica de artéria pulmonar medida pelo ecocardiograma; PAS

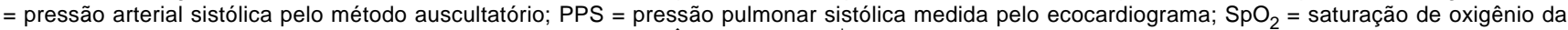
hemoglobina; SVD = sobrecarga do ventrículo direito; $\mathrm{N}=$ normal; $\uparrow$ = aumentada; $\downarrow$ = diminuída. 
Tabela 3 - Medidas realizadas durante cateterismo cardíaco em seis pacientes com hipertensão arterial pulmonar idiopática

\begin{tabular}{|c|c|c|c|c|c|c|c|c|c|c|}
\hline \multirow[t]{2}{*}{ Paciente } & \multirow{2}{*}{$\begin{array}{c}\text { PAD } \\
(\mathrm{mmHg})\end{array}$} & \multirow{2}{*}{$\begin{array}{c}\text { PCP } \\
(\mathrm{mmHg})\end{array}$} & \multirow{2}{*}{$\begin{array}{c}\text { PSAP } \\
(\mathrm{mmHg})\end{array}$} & \multirow{2}{*}{$\begin{array}{c}\text { PMAP } \\
(\mathrm{mmHg})\end{array}$} & \multirow{2}{*}{$\begin{array}{c}\text { PMAo } \\
(\mathrm{mmHg})\end{array}$} & \multirow{2}{*}{$\begin{array}{c}\text { IRP } \\
(\text { dyn.s.cm } \\
-5\end{array}$} & \multirow{2}{*}{$\begin{array}{c}\text { IRS } \\
(\text { dyn.s.cm } \\
-5\end{array}$} & \multicolumn{3}{|c|}{ Reatividade pulmonar } \\
\hline & & & & & & & & ON & $\mathrm{FiO}_{2}=1,0$ & Nifedipina \\
\hline 1 & 13 & 12 & 105 & 75 & 70 & 2800 & 2560 & - & - & - \\
\hline 2 & 08 & 10 & 90 & 70 & 80 & 1289 & 2320 & - & - & - \\
\hline 3 & 12 & 14 & 110 & 85 & 68 & 2800 & 2240 & - & - & - \\
\hline 4 & 15 & 09 & 110 & 73 & 72 & 2640 & 2080 & - & - & - \\
\hline 5 & 14 & 13 & 116 & 77 & 75 & 2080 & 2000 & - & - & - \\
\hline 6 & 13 & 10 & 105 & 80 & 66 & 3200 & 2320 & - & - & - \\
\hline
\end{tabular}

$\mathrm{PAD}=$ pressão átrio direito $(\mathrm{N}=1-5) ; \mathrm{PCP}=$ pressão capilar pulmonar $(\mathrm{N}=2-12)$; PSAP = pressão sistólica de artéria pulmonar ( $\mathrm{N}=30$-35); PMAP = pressão média de artéria pulmonar $(\mathrm{N} \leq 25) ; \mathrm{PMAo}=$ pressão média de aorta; IRP = índice de resistência pulmonar $(\mathrm{N}=80-240)$; IRS = índice de resistência sistêmica $(\mathrm{N}=1600-2400) ; \mathrm{ON}=$ óxido nítrico; $(-)=$ não-responsivo.

Tabela 4 - Resultados e evolução clínica após administração do sildenafil para tratamento de hipertensão arterial pulmonar idiopática

\begin{tabular}{|c|c|c|c|c|c|c|c|c|c|c|c|}
\hline \multirow[t]{2}{*}{ Paciente } & \multicolumn{2}{|c|}{$\begin{array}{c}\text { Classe } \\
\text { funcional }\end{array}$} & \multicolumn{2}{|c|}{$\mathrm{SpO}_{2}$} & \multicolumn{2}{|c|}{$\begin{array}{c}\text { Teste caminhada } \\
6^{\prime}(\mathrm{m})\end{array}$} & \multicolumn{3}{|c|}{ PPS/PAS } & \multirow[t]{2}{*}{ EC } & \multirow{2}{*}{$\begin{array}{c}\text { Tempo de } \\
\text { seguimento } \\
\text { (meses) }\end{array}$} \\
\hline & Antes & Depois & Antes & Depois & Antes & Depois & Antes & Depois & $\%$ & & \\
\hline 1 & IV & II & 90 & 90 & NR & NR & 1,31 & 0,90 & 32 & - & 9 \\
\hline 2 & III & II & 98 & 98 & 260 & 420 & 0,90 & 0,70 & 23 & - & 18 \\
\hline 3 & IV & II & 85 & 90 & NR & NR & 1,22 & 0,90 & 27 & - & 36 \\
\hline $4 *$ & IV & II & 88 & 94 & NR & NR & 1,16 & $1,16 * *$ & 0 & - & 0,3 \\
\hline 5 & III & II & 86 & 92 & 220 & 380 & 1,20 & 1,05 & 13 & - & 7 \\
\hline 6 & IV & III & 74 & 88 & NR & NR & 1,17 & 0,95 & 19 & - & 4 \\
\hline
\end{tabular}

$\mathrm{SpO}_{2}=$ saturação sistêmica de oxigênio; PPS = pressão pulmonar sistólica medida pelo ecocardiograma; PAS = pressão arterial sistólica pelo método auscultatório; $\mathrm{EC}$ = efeitos colaterais; $\mathrm{NR}=$ não realizado, por contra-indicação; $(-)=$ ausente. \% = percentual da redução.

* Morte súbita 1 dia após suspensão do sildenafil sem orientação médica.

** Medida feita 5 dias após início da droga.

(New York Heart Association), diminuição da PPS/PAS, melhora da saturação sistêmica e aumento da distância percorrida no teste da caminhada de 6 minutos. A pressão sistólica pulmonar é geralmente medida pelo ecocardiograma, motivo pelo qual foi usada a relação entre as pressões sistólica pulmonar e sistêmica para comparação. Entretanto, ocorreu óbito de uma paciente de 19 anos (caso 4), internada em classe funcional IV, com saturação sistêmica de $88 \%$ e insuficiência cardíaca grave, que apresentou melhora clínica e aumento da saturação sistêmica. A paciente apresentou morte súbita 24 horas após suspensão inadvertida da medicação enquanto internada, motivo pelo qual todos os pacientes e a equipe envolvida no tratamento devem ser conscientizados para não suspender a medicação abruptamente. Nos demais pacientes, o uso do sildenafil mostrou-se seguro e sem efeitos colaterais, a despeito das doses elevadas. Em quatro pacientes, foi detectada insaturação sistêmica significativa ( $<90 \%$ ), o que não é usual na HAPI. Entretanto, os níveis de pressão arterial pulmonar eram superiores à pressão sistêmica, e a insaturação pode ser explicada por shunt do AD para o átrio esquerdo pelo forame oval permeável, verificado pelo ecocardiograma e pelo cateterismo cardíaco.
A literatura médica tem apresentado um número crescente de relatos de casos sobre o uso do sildenafil oral no tratamento da HP de diversas causas. Tais relatos indicam que a droga beneficia os pacientes na medida em que potencializa ou permite a retirada do óxido nítrico em casos de crises de HP ${ }^{15}$. Associado a outras drogas, o sildenafil pode aumentar a eficácia de cada uma delas, permitindo a redução de suas doses ou o espaçamento de seus interva$\operatorname{los}^{16}$. O sildenafil tem se mostrado igualmente eficaz no tratamento da HP secundária a doenças do colágeno ${ }^{14}$, do tromboembolismo pulmonar crônico sem opção cirúrgica ${ }^{15}$ e, principalmente, no da HAPI 13,17 .

Apesar da melhor compreensão de sua fisiopatologia e das novas opções de tratamento, a HAPI continua sendo uma doença grave, sem cura e sem uma medicação ideal. Todos os métodos terapêuticos disponíveis apresentam restrições, riscos de complicações e são de alto custo. Os pacientes portadores de HAPI em classe funcional IV constituem um grupo especial, já que habitualmente são jovens com uma expectativa de vida menor que 1 ano. Na casuística deste estudo, a despeito do número reduzido de pacientes, observou-se melhora da classe funcional, da tolerância aos esforços (avaliada pelo aumento da distância 
caminhada nos dois casos em condições de realizar o teste da caminhada) e da qualidade de vida, com reintegração social e ausência de internações hospitalares no período de seguimento. Esses resultados sugerem que o sildenafil possa ser uma alternativa terapêutica para esse grupo especial de pacientes. Entretanto, são necessários estudos com inclusão de maior número de pacientes e com tempo de seguimento maior, para que se possam conhecer os resultados a longo prazo e para que se chegue a uma definição das doses e dos intervalos de administração ideais.

\section{Referências}

1. D'Alonzo GE, Barst RJ, Ayres SM, Bergofsky EH, Brundage BH, Detre KM, et al. Survival in patients with pulmonary hypertension: results from a national prospective registry. Ann Intern Med. 1991;115:343-9.

2. Runo JR, Loyd JE. Primary pulmonary hypertension. Lancet. $2003 ; 361: 1533-44$.

3. Farber HW, Loscalzo J. Pulmonary arterial hypertension. N Engl J Med. 2004;351:1655-65.

4. Rich S, Kaufmann E, Levy PS. The effect of high doses of calcium-channel blockers on survival in primary pulmonary hypertension. N Eng J Med. 1992;327:76-81.

5. Morgan JM, McCormack DG, Griffiths MJ. Adenosine as a vasodilator in primary pulmonary hyper tension. Circulation. 1991; 84:1145-9.

6. Jolliet P, Bulpa P, Thorens JB, Ritz M, Chevrolet JC. Nitric oxide and prostacyclin as test agents of vasoreactivity in severe precapillary pulmonary hypertension. Thorax. 1997;52:369-72.

7. Sitbon $\mathrm{O}$, Humbert M, Jagot JL, Taravella O, Fartoukh M, Parent $F$, et al. Inhaled nitric oxide as a screening agent for safely identifying responders to oral calcium channel blockers in primary pulmonary hypertension. Eur Respir J. 1998;12:265-70.

8. Badesch DB, McLaughlin VV, Delcroix M, Vizza CD, Olschewski $\mathrm{H}$, Sitbon $\mathrm{O}$, et al. Prostanoid therapy for pulmonary arterial hypertension. J Am Coll Cardiol. 2004;43(12 Suppl S):S56-61.

9. Channick RN, Sitbon O, Barst RJ, Manes A, Rubin LJ. Endothelin receptor antagonists in pulmonary arterial hypertension. J Am Coll Cardiol. 2004;43 (12 Suppl S):S62-66.
10. Sandoval J, Gaspar J, Pulido T, Bautista E, Martinez-Guerra ML, Zeballos M, et al. Graded balloon dilation atrial septostomy in severe primary pulmonary hypertension: a therapeutic alternative for patients nonresponsive to vasodilator treatment. J Am Coll Cardiol. 1998;32:297-304.

11. Meyers BF, Lynch J, Trulock EP, Guthrie TJ, Cooper JD, Patterson GA. Lung transplantation: a decade experience. Ann Surg. 1999;230:362-71.

12. Ghofrani HA, Schermuly RT, Rose F, Widemann R, Markus G, Kreckel $A$, et al. Sildenafil for long-term treatment of nonoperable chronic thromboembolic pulmonary hypertension. Am J Respir Crit Care Med. 2003;167:1139-41.

13. Michelakis ED, Tymchak W, Noga M, Webster L, Wu XC, Lien D, et al. Long-term treatment with oral sildenafil is safe and improves capacity and hemodynamics in patients with pulmonary arterial hypertension. Circulation. 2003;108:2066-9.

14. Molina J, Luccero E, Luluaga S, Bellonio V, Spindler A, Berman A. Systemic lupus erythematosus associated with pulmonary hypertension: good outcome following sildenafil therapy. Lupus. 2003; 12:321-3.

15. Atz AM, Lefler AK, Fairbrothern DL, Uber WE, Bradley SM. Sildenafil augments the effects of inhaled nitric oxide for postoperative pulmonary hypertension crises. J Thorac Cardiovasc Surg. 2002;124:628-9.

16. Ghofrani HA, Wiedemann R, Rose F, Olschewski $H$, Schermuly RT, Weissmann N, et al. Combination therapy with oral sildenafil and inhaled iloprost for severe pulmonary hypertension. Ann Intern Med. 2002;136:515-22.

17. Humbert M, Sitbon O, Simonneau G. Novel therapeutic perspective in pulmonary arterial hypertension. Eur Respir J. 2003;22:193-4.

Correspondência:

Edmundo Clarindo Oliveira

Rua Teodomiro Cruz, 65/102

CEP 30240-530 - Belo Horizonte, MG

Tel.: (31) 3283.4092

Fax: (31) 3337.9988

E-mail: clarindo@pib.com.br 